\title{
Real Time Inverse Kinematics of Five-Axis CNC Centers
}

\author{
Chu Anh My ${ }^{1(凶)}$, Chi Hieu Le ${ }^{2}$, Xuan Bien Duong ${ }^{1}$, Erik L. \\ J. Bohez ${ }^{3}$, Minh Duc $\mathrm{Vu}^{1}$, Tien Anh Nguyen ${ }^{1}$, Van Cong Nguyen ${ }^{1}$, \\ Michael Parkianather ${ }^{4}$, and Chu Duc Hoang ${ }^{5}$ \\ ${ }^{1}$ Le Quy Don Technical University, Hanoi, Vietnam \\ myca@lqdtu.edu.vn \\ ${ }^{2}$ University of Greenwich, London, UK \\ 3 Asian Institute of Technology, Bangkok, Thailand \\ ${ }^{4}$ Cardiff University, Cardiff CF24 3AA, UK \\ ${ }^{5}$ Ministry of Science and Technology, Hanoi, Vietnam
}

\begin{abstract}
In most of the previous investigations, the kinematics model of fiveaxis computer-numerical control (CNC) centers was formulated just at the position level, and the differential kinematic relationships (velocity, acceleration and jerk of the five joints of a five-axis center) that are necessary for several purposes, especially for investigating the relationship between the limits of a machine's drives and the feed rate maximization (the productivity maximization), have been overlooked. Therefore, this paper addresses the differential kinematic modelling and analysis for the five-axis CNC centers. In particular, the differential kinematic equations are formulated in a parametric domain so that they are useful for investigating the kinematic behaviors of the five-axis centers in real time.
\end{abstract}

Keywords: 5-axis CNC machining $\cdot$ Real time kinematics $\cdot$ Differential kinematics $\cdot$ Inverse kinematics

\section{Introduction}

In the last decades, the high speed five-axis $\mathrm{CNC}$ centers have been widely used to machine complex parts. A five-axis CNC center can be regarded as two cooperative robot arms, one arm carries the cutter and one arm holds the machining part.

In practice, in order to increase the productivity when a five-axis CNC center produces mechanical parts, it is necessary to rise the feed rate which is usually given as a constant in a machining program (G-codes file). When the feed rate of a five-axis $\mathrm{CNC}$ center is increased, the velocity and acceleration of the five drives varies rapidly, especially for that case that a surface having high curvature is machined. Hence, real time analysis and monitoring of velocity and acceleration of the five drives of a fiveaxis CNC center plays an important in optimizing the machining feed rate.

In the literature, several research topics related to 5-axis CNC machining have been taken into account, such as the postprocessor development, the feed rate adjustment, the geometric error compensation, the inverse kinematics, etc. Though there have been 
numerous research works published, few investigations take into account the real time kinematic modelling and analysis for the five-axis CNC centers.

Years ago, a number of papers have been published which focus on the inverse kinematics problem of the five-axis CNC centers [1-18]. The inverse kinematics model of a general five-axis CNC center has been studied in [1-4]. The authors of the works [1-3] generalize the kinematic formulation for a general five-axis CNC center by adding two more extra axes to a common five-axis mechanism, while the authors of [4] used the Screw Theory to formulate the kinematics model of a five-axis CNC machine. The kinematic modelling and analysis for a class of five-axis CNC center of which the rotational axes are not orthogonal was well documented [5-9]. The works [5, 6] studied the inverse kinematic model of the five-axis CNC center DMU 50e. Other types of fiveaxis CNC centers were investigated in [7-9]. In the same context, the kinematic model of the class of five-axis CNC center with two orthogonal rotational axes was formulated. Three main types of this machine class were investigated in $[10,11]$. The kinematic solution of some individual five-axis CNC centers like Maho 600e and Spinner U5-620 was presented in [12-18].

Obviously, though there have been several research works related to the inverse kinematics of the five-axis CNC centers, none of them takes into account the real time inverse kinematic modelling and analysis for a better kinematic performance analysis of the five-axis CNC centers. In this paper, a novel real time kinematic modelling and analysis of the five-axis CNC centers is presented. It has shown that the method proposed in this paper is useful for analysis and monitoring the kinematic performances of the five drives of any five-axis CNC center.

\section{Real Time Kinematic Modelling of a General Five-Axis CNC Center}

Based on the kinematic modelling of a general five-axis CNC center presented in our previous work [14], the inverse kinematic equations at differential levels can be formulated in a time domain, and the derivation of these equations are presented in this section. As shown in [14], the forward kinematic equation of a general five-axis CNC center was given as

$$
\boldsymbol{X}=\boldsymbol{f}(\boldsymbol{q})
$$

where $\boldsymbol{X}=\left[\begin{array}{lllll}x & y & z & \phi & \varphi\end{array}\right]^{T}$ is the pose of the cutter, $\boldsymbol{q}=\left[\begin{array}{lllll}q_{1} & q_{2} & q_{3} & q_{4} & q_{5}\end{array}\right]^{T}$ are the five axes of a five-axis CNC center.

Suppose that $f(t)=\dot{s}(t)$ is the velocity of the cutter along the tool path $\boldsymbol{X}(u)=\left[\begin{array}{lllll}x(u) & y(u) & z(u) & \phi(u) & \varphi(u)\end{array}\right]^{T} \in \Re^{5}, u \in[0,1]$, in which $s$ is the length of a cutting path, and $\mathrm{t}$ is time. The inverse kinematic solution can be written as follows:

$$
q=f^{-1}(X)
$$


From Eq. (1), the following derivative can be yields

$$
\dot{\boldsymbol{X}}=J \dot{\boldsymbol{q}}
$$

Note that $\boldsymbol{J}=\frac{\partial \boldsymbol{f}}{\partial \boldsymbol{q}}$ is denoted for the Jacobian matrix. Based on Eq. (3), the velocity of the joints can be calculated as:

$$
\dot{\boldsymbol{q}}=\boldsymbol{J}^{-1} \dot{\boldsymbol{X}}=\boldsymbol{J}^{-1} \frac{d \boldsymbol{X}}{d s} \frac{d s}{d t}=\boldsymbol{J}^{-1} \boldsymbol{X}_{s}^{\prime} \dot{s}
$$

Note that

$$
\begin{gathered}
d s \simeq\left|\frac{d \boldsymbol{X}}{d u}\right| d u \\
\boldsymbol{X}_{s}^{\prime}=\frac{d \boldsymbol{X}}{d s}=\frac{d \boldsymbol{X} / d u}{d s / d u}=\frac{d \boldsymbol{X} / d u}{|d \boldsymbol{X} / d u|}=\frac{\boldsymbol{X}_{u}^{\prime}}{\left|\boldsymbol{X}_{u}^{\prime}\right|}
\end{gathered}
$$

Thus we obtain the velocity as follows:

$$
\dot{\boldsymbol{q}}=\boldsymbol{J}^{-1} \frac{\boldsymbol{X}_{u}^{\prime}}{\left|\boldsymbol{X}_{u}^{\prime}\right|} f
$$

Equation (7) implies that at any value of time, the velocities of the five axes of a given five-axis CNC center can be computed in time domain with respect to the geometric properties of the prescribed cutting curve $\mathbf{X}_{u}^{\prime}$, and the Jacobian $\mathbf{J}$ of a five-axis CNC center. From Eq. (3) we can yield

$$
\ddot{\boldsymbol{X}}=J \ddot{\boldsymbol{q}}+\dot{J} \dot{\boldsymbol{q}}
$$

Hence

$$
\ddot{\boldsymbol{q}}=\boldsymbol{J}^{-1}(\ddot{\boldsymbol{X}}-\dot{\boldsymbol{J}} \dot{\boldsymbol{q}})
$$

Note that

$$
\begin{gathered}
\dot{\boldsymbol{X}}=\boldsymbol{X}_{s}^{\prime} \dot{s} \\
\ddot{\boldsymbol{X}}=\frac{d \boldsymbol{X}_{s}^{\prime}}{d s} \dot{s}^{2}+\boldsymbol{X}_{s}^{\prime} \ddot{s}=\boldsymbol{X}_{s}^{\prime \prime} \dot{s}^{2}+\boldsymbol{X}_{s}^{\prime} \ddot{s}
\end{gathered}
$$

Based on Eq. (5), Eq. (11) is obtained

$$
s_{u}^{\prime}=\left|\boldsymbol{X}_{u^{\prime}}^{\prime}\right|
$$


Equation (6) now becomes

$$
\boldsymbol{X}_{s}^{\prime}=\frac{\boldsymbol{X}_{u}^{\prime}}{s_{u}^{\prime}}
$$

Equation (12) can be obtained as follows:

$$
\boldsymbol{X}_{s}^{\prime \prime}=\frac{\left(d \boldsymbol{X}_{u}^{\prime} / d s\right) s_{u}^{\prime}+\boldsymbol{X}_{u}^{\prime}\left(d s_{u}^{\prime} / d s\right)}{\left|\boldsymbol{X}_{u}^{\prime}\right|^{2}}
$$

It is also noted that $\frac{d \boldsymbol{X}_{u}^{\prime}}{d s}=\frac{\boldsymbol{X}_{u}^{\prime \prime}}{s_{u}^{\prime}}=\frac{\boldsymbol{X}_{u}^{\prime \prime}}{\left|\boldsymbol{X}_{u}^{\prime}\right|} ; \frac{d s_{u}^{\prime}}{d s}=\frac{s_{u}^{\prime \prime}}{s_{u}^{\prime}}=\frac{\left(\boldsymbol{X}_{u}^{\prime}\right)^{T} \boldsymbol{X}_{u}^{\prime \prime}}{\left|\boldsymbol{X}_{u}^{\prime}\right|^{2}}$

Hence

$$
\boldsymbol{X}_{s}^{\prime \prime}=\frac{\boldsymbol{X}_{u}^{\prime \prime}}{\left|\boldsymbol{X}_{u}^{\prime}\right|^{2}}+\frac{\boldsymbol{X}_{u}^{\prime}\left(\left(\boldsymbol{X}_{u}^{\prime}\right)^{T} \boldsymbol{X}_{u}^{\prime \prime}\right)}{\left|\boldsymbol{X}_{u}^{\prime}\right|^{4}}
$$

Now Eq. (9) becomes

$$
\ddot{\boldsymbol{q}}=\boldsymbol{J}^{-1}\left(\left(\frac{\boldsymbol{X}_{u}^{\prime \prime}}{\left|\boldsymbol{X}_{u}^{\prime}\right|^{2}}+\frac{\boldsymbol{X}_{u}^{\prime}\left(\left(\boldsymbol{X}_{u}^{\prime}\right)^{T} \boldsymbol{X}_{u}^{\prime \prime}\right)}{\left|\boldsymbol{X}_{u}^{\prime}\right|^{4}}\right) f^{2}+\frac{\boldsymbol{X}_{u}^{\prime}}{\left|\boldsymbol{X}_{u}^{\prime}\right|} \dot{f}-\dot{\boldsymbol{J}} \dot{\boldsymbol{q}}\right)
$$

Equation (14) is the joint accelerations of a five-axis CNC center. In order to calculate the jerk of the joints, taking time derivative of Eq. (1) yields

$$
\dddot{\boldsymbol{X}}=\dddot{J \boldsymbol{q}}+2 \dot{\boldsymbol{J}} \ddot{\boldsymbol{q}}+\ddot{\boldsymbol{J}} \dot{\boldsymbol{q}}
$$

Hence

$$
\dddot{\boldsymbol{q}}=\boldsymbol{J}^{-1}(\dddot{\boldsymbol{X}}-2 \dot{\boldsymbol{J}} \ddot{\boldsymbol{q}}-\ddot{\boldsymbol{J}} \dot{\boldsymbol{q}})
$$

We calculate

$$
\begin{gathered}
\dddot{\boldsymbol{X}}=\frac{d \ddot{\boldsymbol{X}}}{d s} \dot{s}=\frac{d}{d s}\left(\boldsymbol{X}_{s}^{\prime \prime} \dot{s}^{2}+\boldsymbol{X}_{s}^{\prime} \ddot{s}\right) \dot{s}=\left(\boldsymbol{X}_{s}^{\prime \prime \prime} \dot{s}^{2}+\boldsymbol{X}_{s}^{\prime \prime} \ddot{s}\right) \dot{s} \\
\boldsymbol{X}_{s}^{\prime \prime \prime}=\frac{d \boldsymbol{X}_{s}^{\prime \prime}}{d u} \frac{1}{\left|\boldsymbol{X}_{u}^{\prime}\right|} \\
=\frac{\boldsymbol{X}_{u}^{\prime \prime \prime}}{\left|\boldsymbol{X}_{u}^{\prime}\right|^{3}}+\frac{\boldsymbol{X}_{u}^{\prime \prime}\left[\left(\boldsymbol{X}_{u}^{\prime}\right)^{T} \boldsymbol{X}_{u}^{\prime \prime}\right]+\boldsymbol{X}_{u}^{\prime}\left[\left(\boldsymbol{X}_{u}^{\prime \prime}\right)^{T} \boldsymbol{X}_{u}^{\prime \prime}+\left(\boldsymbol{X}_{u}^{\prime}\right)^{T} \boldsymbol{X}_{u}^{\prime \prime \prime}\right]}{\left|\boldsymbol{X}_{u}^{\prime}\right|^{5}}
\end{gathered}
$$


Finally, Eq. (16) becomes

$$
\ldots=\boldsymbol{J}^{-1}\left\{\begin{array}{c}
\frac{\boldsymbol{X}_{u}^{\prime \prime \prime}}{\left|\boldsymbol{X}_{u}^{\prime}\right|^{3}} f^{3}+\frac{\boldsymbol{X}_{u}^{\prime \prime}\left[\left(\boldsymbol{X}_{u}^{\prime}\right)^{T} \boldsymbol{X}_{u}^{\prime \prime}\right]+\boldsymbol{X}_{u}^{\prime}\left[\left(\boldsymbol{X}_{u}^{\prime \prime}\right)^{T} \boldsymbol{X}_{u}^{\prime \prime}+\left(\boldsymbol{X}_{u}^{\prime}\right)^{T} \boldsymbol{X}_{u}^{\prime \prime \prime}\right]}{\left|\boldsymbol{X}_{u}^{\prime}\right|^{5}} f^{3}+ \\
{\left[\frac{\boldsymbol{x}_{u}^{\prime \prime}}{\left|\boldsymbol{X}_{u}^{\prime}\right|^{2}}+\frac{\boldsymbol{x}_{u}^{\prime}\left(\left(\boldsymbol{x}_{u}^{\prime}\right)^{T} \boldsymbol{X}_{u}^{\prime \prime}\right)}{\left|\boldsymbol{X}_{u}^{\prime}\right|^{4}}\right] \dot{f} f-2 \dot{\boldsymbol{J}} \ddot{\boldsymbol{q}}-\ddot{\boldsymbol{J}} \dot{\boldsymbol{q}}}
\end{array}\right\}
$$

Note that $\dot{\boldsymbol{J}}$ and $\square \boldsymbol{J}$ are formulated as follows: $\dot{\boldsymbol{J}}=\left[\frac{\partial J_{i j}}{\partial \boldsymbol{q}}\right] \otimes \dot{\boldsymbol{q}}$ and

$$
\ddot{\boldsymbol{J}}=\left[\frac{\partial}{\partial \boldsymbol{q}}\left(\left[\frac{\partial J_{i j}}{\partial \boldsymbol{q}}\right] \otimes \dot{\boldsymbol{q}}\right)\right] \otimes\left[\begin{array}{c}
\dot{\boldsymbol{q}} \\
\cdots \\
\dot{\boldsymbol{q}}
\end{array}\right]_{1 \times 5}+\left[\frac{\partial J_{i j}}{\partial \boldsymbol{q}}\right] \otimes \ddot{\boldsymbol{q}}
$$

Equations (7), (14), (19) are the vectors of velocities, accelerations and jerks of the five drives of a five-axis $\mathrm{CNC}$ center that are calculated in time domain. Given an individual five-axis $\mathrm{CNC}$ center, the value of this vector can be computed with respect to the velocity of cutter along a given tool path.

\section{Numerical Analysis of Real Time Inverse Kinematics for Some Five-Axis CNC Centers}

Example 1. The velocities and accelerations of the five drives of the five-axis CNC center DMU 50e

The programmed tool path and the velocity of the cutter are given in Fig. 2.

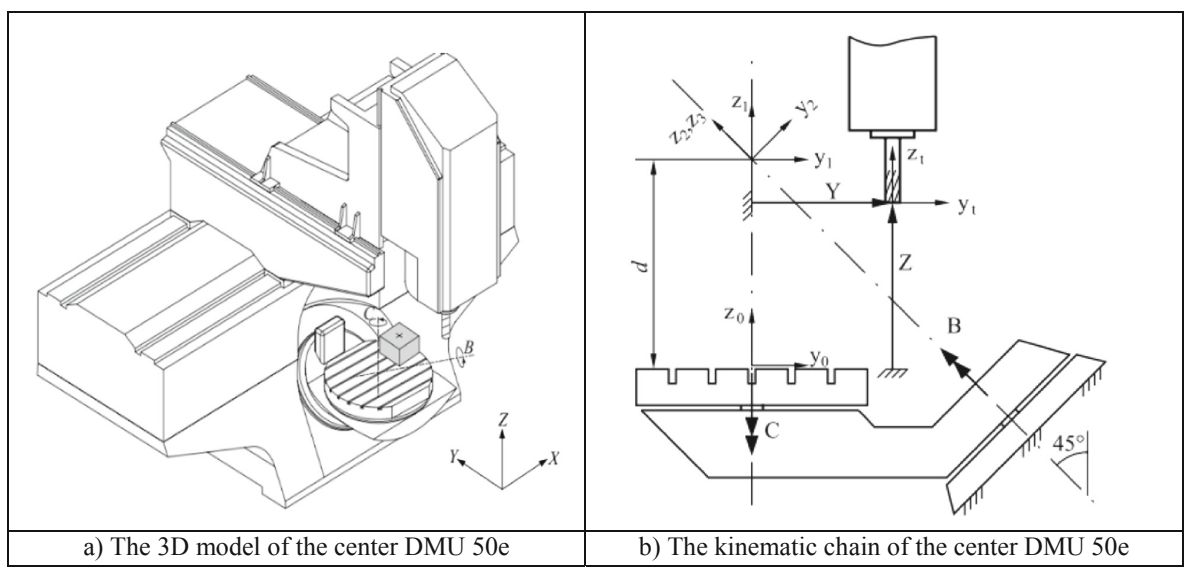

Fig. 1. Five-axis CNC center DMU 50e 


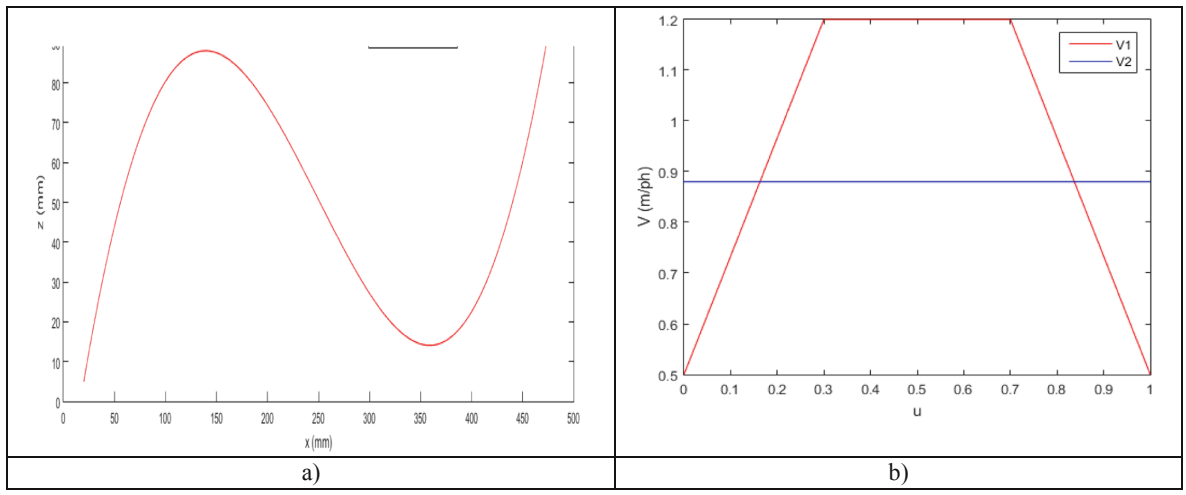

Fig. 2. a) A given tool path and b) the prescribed velocity profile of the cutter

Let's consider the model and the kinematic chain of the five-axis CNC center DMU 50e as shown in Fig. 1. Using Eqs. (7), (14), (19) to calculated the real time inverse kinematic solution for the five-axis CNC center DMU 50e, the velocities and accelerations of the machine axes are obtained and presented in the following Figs. 3 and 4.

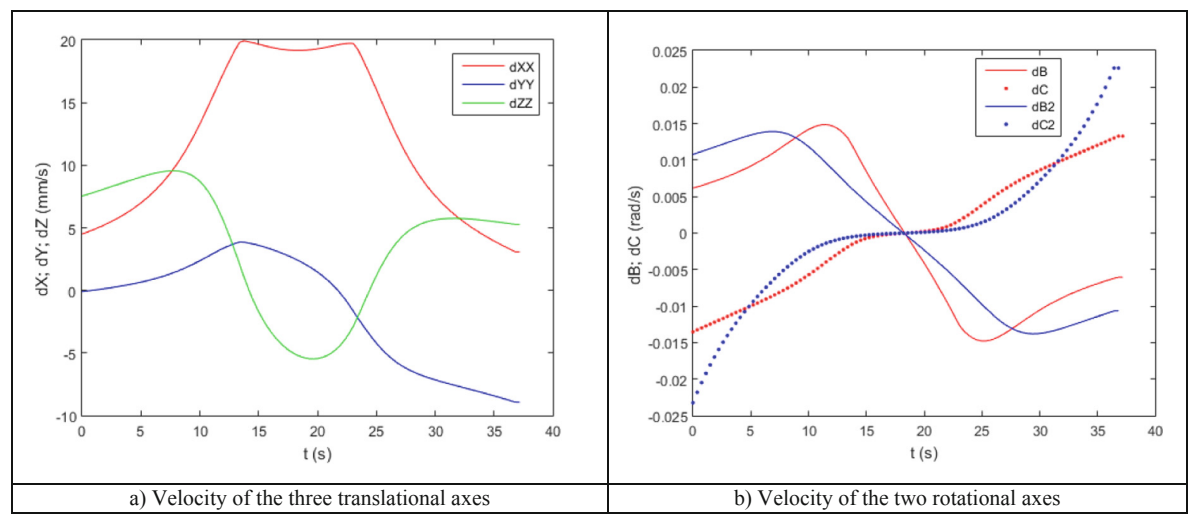

Fig. 3. The velocities of the five axes 


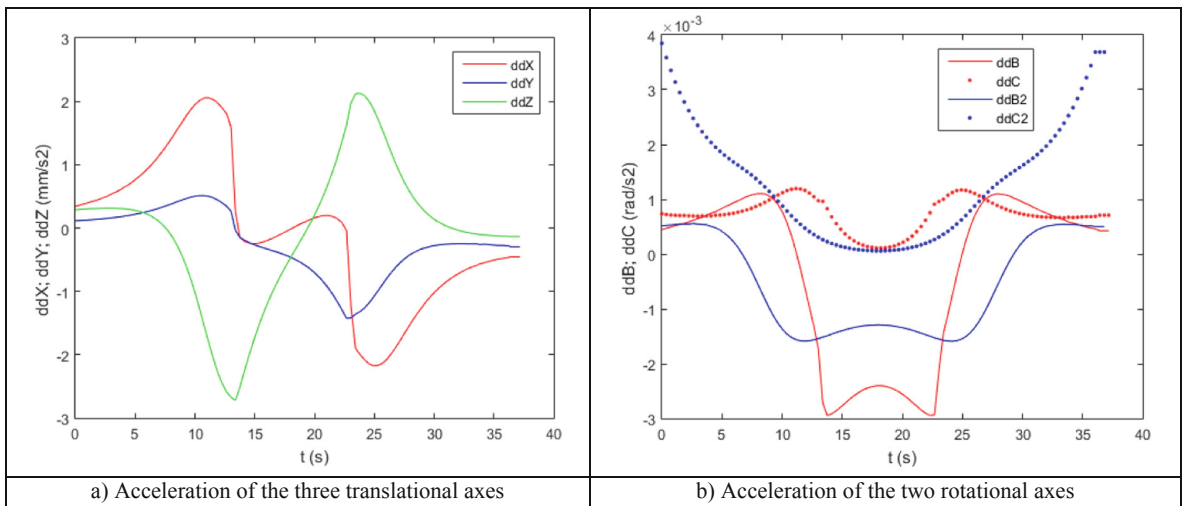

Fig. 4. The accelerations of the five axes

Example 2. The velocities and accelerations of the five drives of the five-axis CNC center Spinner U5-620

Figure 5 shows the 3D model and the kinematic chain of the five-axis CNC center Spinner U5-620. The velocities and accelerations of the exes of the machine are computed and shown in Figs. 6 and 7, respectively.

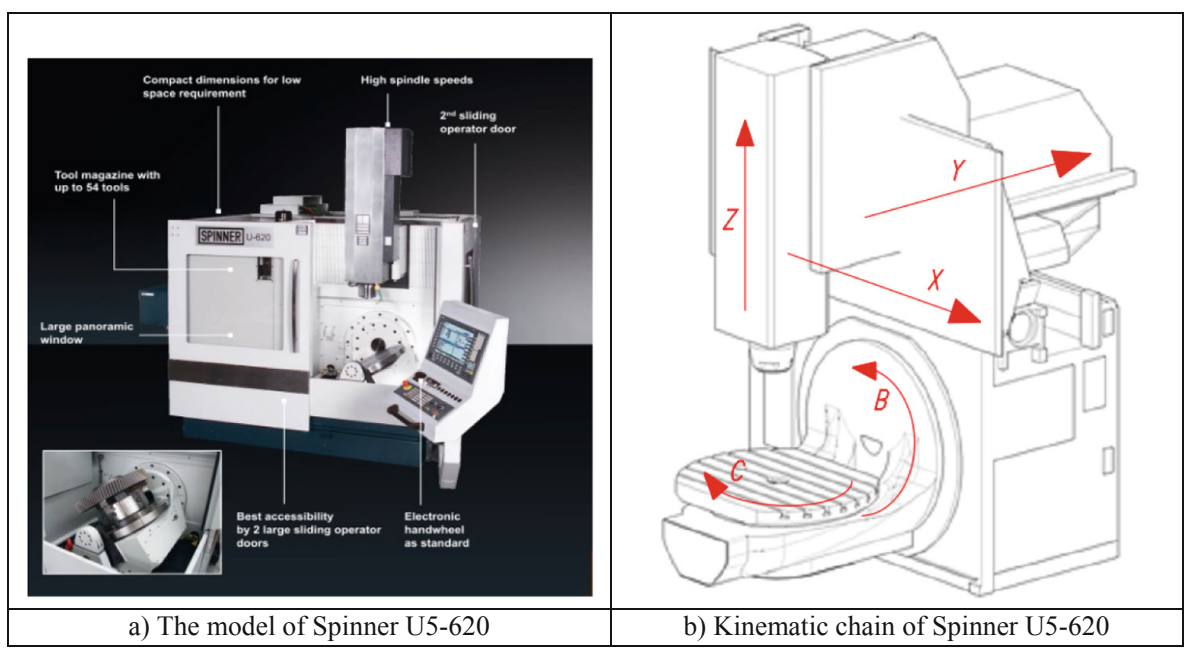

Fig. 5. The five-axis CNC center DMU 50e 


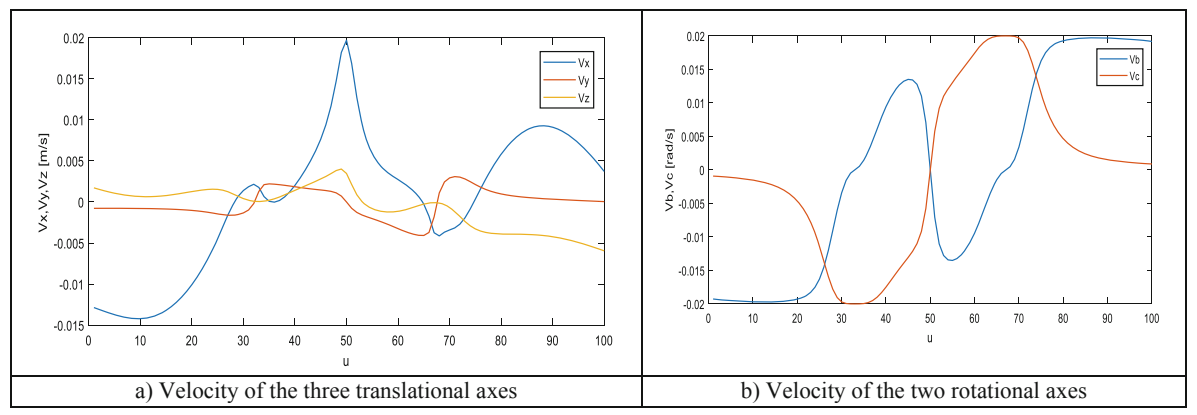

Fig. 6. The velocities of the five axes

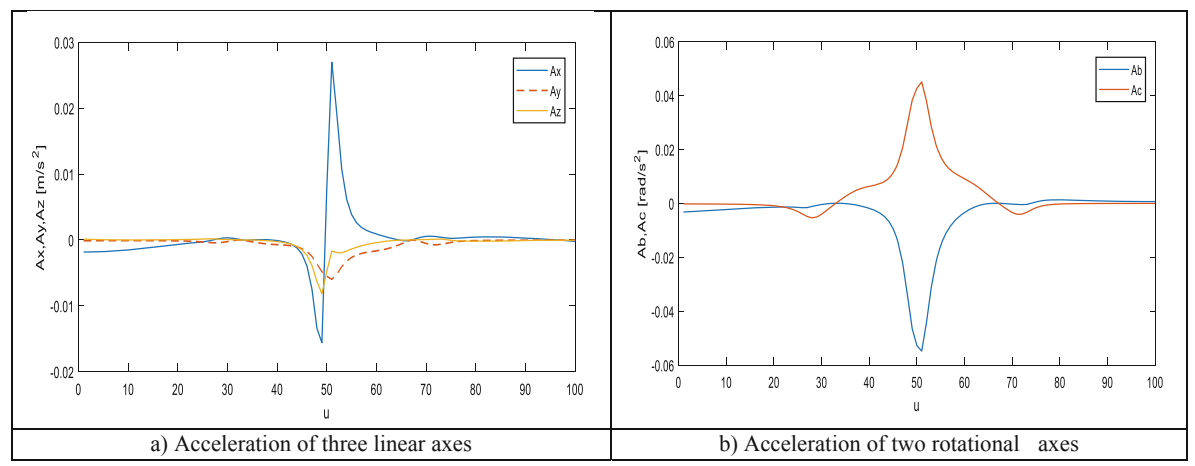

Fig. 7. The accelerations of the five axes

\section{Conclusion}

The kinematics model of the five-axis CNC centers was successfully formulated in the time domain. Note that the inverse kinematic equations yielded in this study are useful to investigate the kinematic behavior of the machine's drives in real time. The numerical example shows the effectiveness of the proposed formulation. The real time kinematic modelling of the five-axis CNC centers presented in this paper can be extended for some future works, e.g. the feed rate optimization, the postprocessor development, the drive capability monitoring, etc.

Acknowledgement. This research was funded by the UK Department for Business, Energy and Industrial Strategy and delivered by the British Council, under a Newton Fund Project ID 528085858 . 


\section{References}

1. Tutunea-Fatan, F.H.Y.: Configuration analysis of five-axis machine tools using a generic kinematic model. Int. J. Mach. Tools Manuf. 44, 1235-1243 (2004)

2. Yang, J., Altintas, Y.: Generalized kinematics of five-axis serial machines with non-singular tool path generation. Int. J. Mach. Tools Manuf 75, 119-132 (2013)

3. She, C.H., Chang, C.C.: Design of a generic five-axis postprocessor based on generalized kinematics model of machine tool. Int. J. Mach. Tools Manuf 47, 537-545 (2007)

4. My, C.A., Bohez, E.L.: A novel differential kinematics model to compare the kinematic performances of five-axis CNC centers. Int. J. Mech. Sci. 163, 105117 (2019)

5. My, C.A.: Integration of CAM systems into multi-axes computerized numerical control machines. In: 2010 IEEE Second International Conference on Knowledge and Systems Engineering, 119-124 (2010)

6. My, C.A., Bohez, E.L.: New algorithm to minimise kinematic tool path errors around 5-axis machining singular points. Int. J. Prod. Res. 54, 5965-5975 (2016)

7. My, C.A., Hai, V.X.: Generalized pseudo inverse kinematics at singularities for developing five-axes CNC machine tool postprocessor. Vietnam J. Mech. 35, 147-155 (2013)

8. Sørby, K.: Inverse kinematics of five-axis machines near singular configurations. Int. J. Mach. Tools Manuf 47, 299-306 (2007)

9. She, C.H., Huang, Z.T.: Postprocessor development of a five-axis machine tool with nutating head and table configuration. Int. J. Adv. Manuf. Technol. 38, 728-740 (2008)

10. Lee, R.S., She, C.H.: Developing a postprocessor for three types of five-axis machine tools. Int. J. Adv. Manuf. Technol. 13, 658-665 (1997)

11. Xu, H.Y., Hu, L.A., Hon-Yuen, S.K., Xu, L.A.: A novel kinematic model for five-axis machine tools and its CNC applications. Int. J. Adv. Manuf. Technol. 67, 1297-307 (2013)

12. Farouki, R.T., Han, C.Y., Li, S.: Inverse kinematics for optimal tool orientation control in 5axis CNC machining. Comput. Aided Geometric Des. 31, 13-26 (2014)

13. Boz, Y., Lazoglu, I.: A postprocessor for table-tilting type five-axis machine tool based on generalized kinematics with variable feedrate implementation. Int. J. Adv. Manuf. Technol. 66, 1285-1293 (2013)

14. My, C.A., Bohez, E.L.: Multi-criteria optimization approach for 5-axis $\mathrm{CNC}$ tool path planning: modeling methodology. In Proceedings of the 3rd Asian Conference on Industrial Automation and Robotics, pp. 5-11 (2003)

15. My, C.A., Bohez, E.L., Makhanov, S.S., Munlinb, M., Phien, H.N., Tabucanon, M.T.: On 5axis freeform surface machining optimization: vector field clustering approach. Int. J. CAD/CAM 5, 1-11 (2009)

16. My, C.A., Bohez, E. L. J., Makhanov, S.S.: Critical point analysis of 3D vector field for 5axis tool path optimization. In: Proceedings of the 4th Asian Conference on Industrial Automation and Robotics, ACIAR, pp. 11-13 (2005)

17. Bohez, E.L.J.: Five-axis milling machine tool kinematic chain design and analysis. Int. J. Mach. Tools Manuf 42, 505-520 (2002)

18. My, C.A., Bien, D.X., Tung, B.H., Van Cong, N.: New feed rate optimization formulation in a parametric domain for 5-axis milling robots. In: International Conference on Computer Science, Applied Mathematics and Applications, pp. 403-411 (2019) 